\title{
CRAT: A Novel Routing Algorithm for Wireless Sensor Networks Based on Cooperative MIMO
}

\author{
Pingyuan Liang ${ }^{1,2}$ and Xingcheng Liu ${ }^{1}$ \\ ${ }^{1}$ School of Information Science and Technology \\ Sun Yat-sen University, Guangzhou, China \\ ${ }^{2}$ College of Physics Science and Information Engineering \\ Jishou University, Jishou, China \\ liangpingyuan123@163.com \\ isslxc@mail.sysu.edu.cn
}

\begin{abstract}
Cooperative MIMO technology can be used to reduce the total energy consumption compared with traditional SISO technology under condition that the transmission distances surpass a threshold in wireless sensor networks. In order to solve the routing optimization problem of multi-hop extensible wireless sensor networks based on cooperative MIMO, a novel cost-based routing algorithm with thresholds (CRAT) is proposed. In the algorithm, the routing paths stretch out unboundedly from monitor node and reach the base station ultimately fixed on thresholds, and the routing structure of the CRAT algorithm is different from other cost-based routing algorithms. The energy consumption and thresholds of the algorithm have been analyzed. After that, we define the cost function of CRAT algorithm according to relevant parameters including energy consumption, residual energy, hops and antennas. Finally, the experiments verify the effectiveness of the CRAT algorithm in saving energy, and the reasonableness of the cost function.
\end{abstract}

Keywords-Wireless Sensor Networks; Cooperative MIMO; Energy Consumption; Routing Algorithm; CRAT;

\section{INTRODUCTION}

In wireless sensor networks (WSN), thousands and thousands of small-size and low-cost sensor nodes are deployed in a large area randomly, and they are capable of collecting and disseminating environmental information at anytime, in anywhere, under any circumstances. A self-organization network will be constructed when the collected data want to be sent to a sink or base station (BS) through multiple hops [1]. To replace the batteries of vast nodes is difficult, so energy efficiency is necessary for WSN. Cooperative Multi-Input Multi-Output (MIMO) technology can save a quantity of energy compared with traditional Single-Input Single-Output (SISO) technology $[2,3]$. The cluster head $(\mathrm{CH})$ will consume much more energy than other nodes, because the $\mathrm{CHs}$ have to receive, encode and forward the data packets in cooperative MIMO transmission. Therefore, in order to prolong the lifetime of WSN and enhance the sensing coverage, saving more energy and balancing energy consumption are key factors to design routing algorithms [4].

F. Ye et al. [5] studied a scalable solution about delivering messages from any sensor nodes to BS along the path with minimum cost in WSN. The straightforward solution to build the field was flooding, while the flooding-based design suffered excessive advertisement messages (ADM). Therefore, a back-off algorithm was proposed to decrease the ADM. In the routing algorithm proposed in this paper, the packet with cost value of a node would be replaced when a new packet with smaller cost value arrives, so the minimum cost value getting to BS from any node would be saved at the end of the phase of routing building. D. Yuan et al. [6] proposed a routing protocol CEERP, in which each sensor node found an appropriate path to forward data packets through calculating and comparing the cost values of the relative cost-functions. Compared with the work of Direct, LEACH and PEGASIS on energy consumption and lifetime, the results showed that CEERP outperformed the existing methods in maximizing the network lifetime with less latency. In [7], an energy consumption model was developed to investigate the energy saving performance by incorporating the cooperative MIMO scheme with the multi-hop WSN. Then, an optimization model was developed to find the optimal number of virtual nodes and the optimal number of hops. However, the cost-based routing protocol only considered about the energy consumption in its cost functions. In [8], a multihop Multi-Input Single-Output (MISO) WSN model was built. In the cross-layer design, the cost-based routing algorithm was integrated with throughput, delay and optimized energy consumption. However, when many factors were considered, the protocol became very complicated. These design ideas of the above papers were the same in replacing the cost function values, and they all initiated the routing from BS.

In this paper, a new cost-based routing algorithm with thresholds (CRAT) is proposed. Compared with those costbased routing mentioned above, the CRAT algorithm has the following differences and superiorities. Firstly, in order to save more energy, the distance thresholds are considered in cooperative MIMO transmission, and more energy can be saved statistically compared with other routing algorithms without threshold. Secondly, the routing structure of the CRAT algorithm differs from other algorithms. The CRAT algorithm builds the routing paths from monitor node (MN) instead of BS. The CHs forward the packet with minimum cost value directly in each hop, and only the BS needs to compare the cost function values transmitted from multiple paths. Thirdly, 
energy consumption balances are realized by building $\mathrm{CHs}$ framework dynamically, and many sensor nodes located in the gaps of clusters will not consume energy. Therefore, more energy will be saved than the previous algorithms. Finally, various factors including energy consumption, residual energy, hops, antennas of transmitters and receivers are considered in the cost function.

The rest of this paper is organized as follows. In Section II, we build the multi-hop WSN system model. The CRAT algorithm, which includes three phases (i.e., routing building, data transmission and routing maintenance), is proposed in Section III. In Section IV, according to the system model and the CRAT algorithm, the total energy consumption and the distance threshold are analyzed. Combining with the residual energy, hops and antennas, we define the cost function. In section $\mathrm{V}$, the reasonableness and effectiveness of CRAT algorithm are verified in simulations. Finally, Section VI concludes the paper with the future works.

\section{SYSTEM MODEL}

We can make the following assumption:

1) All nodes are isomorphic, and each of them has a unique ID.

2) The transmission distances are approximations under condition that channel state information (CSI) and bit error rate $(\mathrm{BER})$ are known.

3) The energy and complexity of the BS are not limited.

4) The node-to-node transmission can be divided in two kinds: inter-cluster long transmission (ILT) and intra-cluster short transmission (IST) [2].

The multi-hop WSN system model is shown in Fig. 1, where the data collected by MN will be transmitted to a remote BS by multiple hops. During the transmission, some of these sensor nodes will be organized into multiple clusters. Each cluster has only one $\mathrm{CH}$, and the $\mathrm{CHs}$ are in the middle of the clusters. Cooperative MIMO transmission happens between the cluster and the next adjacent cluster on the routing path to BS. The $\mathrm{CH}$ and cooperative nodes (CNs) in a cluster encode and transmit the data packets to the next cluster according to the orthogonal space time block code (STBC). When an instant event happens, the $\mathrm{MN}$ collects data and searches a $\mathrm{CH}$ around, and the $\mathrm{CH}$ searches new $\mathrm{CHs}$ around in steps limited by ILT threshold again, and so on. Until all CHs have been built, the CHs around BS will find BS ultimately. The paths from $\mathrm{MN}$ to the BS can be regarded as a routing tree which grows unlimitedly. Since the location of $\mathrm{MN}$ and $\mathrm{CHs}$ on the paths change randomly, the energy consumption can be kept in balance. The structure of routing tree is shown in Fig. 2 . After the location of $\mathrm{MN}$ and the $\mathrm{CH}$ framework have been fixed on, there are multiple paths from MN to BS. During the multi-hop transmission, the cost value of each $\mathrm{CH}$ will be minimized along to BS. Unlike the algorithms in [5-8], the back-off algorithm is not necessary to obtain the minimum cost value. Of course, since there are a few paths getting to $\mathrm{BS}$, the cost function value will be calculated and compared, and BS will choose the routing path with minimum cost value.

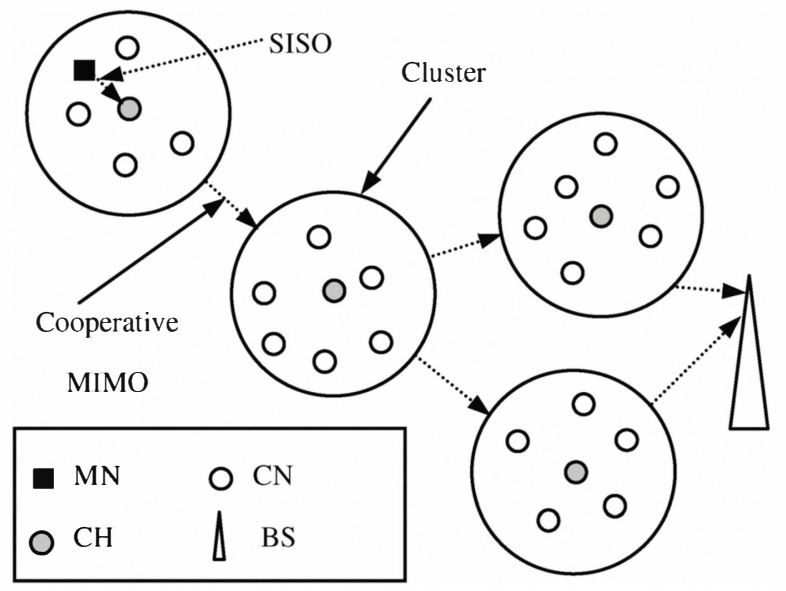

Fig. 1. Multi-hop WSN system model

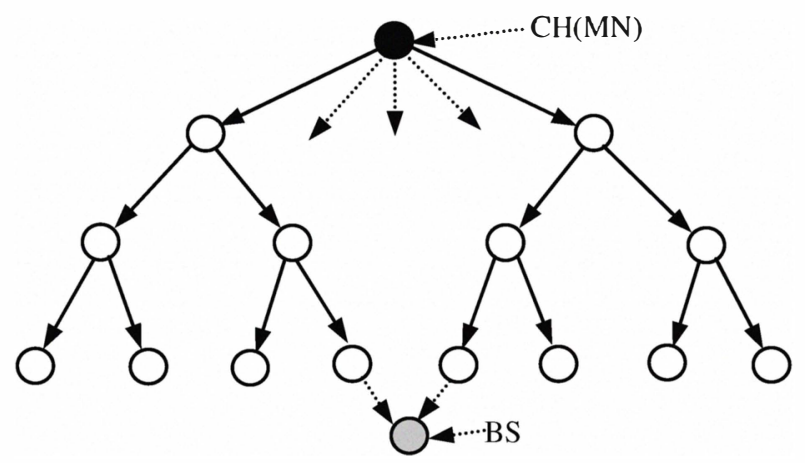

Fig. 2. Structure of routing tree

\section{CRAT ALGORITHM}

The CRAT algorithm can be divided into three phases: routing building, data transmission and routing maintenance. We firstly assume the following aspects:

1) All sensor nodes have the same initial energy $E_{0}$. When the $i^{\text {th }}$ data forwarding cycle begins, the residual energy of each node is defined as $E_{i}$. The residual energy threshold of $\mathrm{CH}$ is defined as $E_{t h}$, and all nodes whose energy is lower than $E_{t h}$ cannot be CHs. $E_{T_{-} j}$ and $E_{R_{-} j}$ are the residual energy levels of transmitters and receivers in each hop respectively, they are determined by $E_{i}$ of the nodes. $N_{T_{-} j}$ and $N_{R_{-j}}$ are the total number of antennas (i.e., they include the number of $\mathrm{CH}$ and $\mathrm{CNs}$ ) of transmitter and receiver respectively.

2) The threshold of ILT distance is defined as $d_{l t h}$, and the threshold of IST distance is defined as $d_{s t h}$. The maximum ILT distance in the $i^{t h}$ data forwarding cycle is defined as $d_{i}$.

3) The routing table of the transmitter $\mathrm{CH}$ in the $j^{\text {th }}$ hop includes following information: ID routing order table; the cost value $C_{T_{-} j}$ from $\mathrm{MN}$ to the $\mathrm{CH}$ of the former $(j-1)^{t h}$ hop; the antennas $N_{T_{-} j}$ of transmitter around $\mathrm{CH}_{\text {; the }} E_{T_{-} j}$ and the IST average distance $d_{\bar{m}_{-} T_{-} j}$ of transmitter. The antennas $N_{R_{-} j}$ of the receiver; the IST average distance $d_{\bar{m}_{-} R_{-} j}$ of receiver; hops $j$ and $E_{R_{-} j}$ (The relations of these parameters will be analyzed 
in part IV). All initial values of these parameters are defined as 0 in the routing table.

\section{A. The Phase of Routing Building}

In the phase of routing building, the $\mathrm{CH}$ framework is fixed on dynamically according to thresholds of distances and residual energy in order to save more energy and balance the energy consumption among the sensor nodes. The cost function value $C_{R_{-} j}$ will be calculated according to all relative parameters, and the new parameters obtained just now will be transmitted to the next $\mathrm{CH}$. Each $\mathrm{CH}$ will select eligible $\mathrm{CNs}$ to transmit in cooperative MIMO technology, where the nodes having better CSI will be CNs preferentially. Finally, the BS will choose the path with minimum cost value to transmit. The process of routing building can be described as follows.

Step 1: When a node finds that something happens, it becomes a $\mathrm{MN}$ and detects the residual energy itself. Case 0: if $E_{i}>E_{t h}$, then marks itself as a $\mathrm{CH}$, and detects $N_{T_{-} j}$ $(j=0)$ and $d_{\bar{m}_{-} T_{-} j}(j=0)$; Case 1: the MN sends a near $\mathrm{CH}$ request (NCHR) message in a manner of IST to the nodes around the MN.

Step 2: When the nodes around MN receive NCHR message, they detect their residual energy. Case 0 : if $E_{i}<E_{t h}$ or $d>d_{s t h}$, then sleep; Case 1: if $E_{i}>E_{t h}$ and $d<d_{s t h}$, then wait for a random time $T_{R}$.

Step 3: After the Case 1 in Step 2 or Step 6, in the period of $T_{R}$, one of the three cases will happen. Case 10: if None NCHAM is received, then they broadcast NCHAM message; Case 11: if 1 NCHAM is received, then the nodes return belonging message (BM); Case 12: if two or more NCHAM are received, then they return none of $\mathrm{BM}$, and abstain from being $\mathrm{CH}$.

Step 4: After a node sends NCHAM, if the BM itself is returned, then marks itself as a $\mathrm{CH}$. Otherwise, it waits for $T_{R}(\tau=0,1,2, \ldots 7)$. If none of BM is returned until $\tau=7$, then marks itself as an isolated $\mathrm{CH}$.

Step 5: The new CH detects $j, N_{R_{-} j}, d_{j}, d_{\bar{m}_{-} R_{-} j}$ and $E_{R_{-} j}$, Connected with $C_{T_{-} j}, N_{T_{-} j}, d_{\bar{m}_{-} T_{-} j}$ and $E_{T_{-} j}$ messages carried by NCHR or FCHR, it can calculate the new routing cost value $C_{R_{-} j}$ from $\mathrm{MN}$ to $\mathrm{CH}$.

Step 6: The new $\mathrm{CHs}$ send far $\mathrm{CH}$ request (FCHR) messages in ILT manner. Farther nodes receive the FCHR, Case 0: if $d_{l t h}<d<d_{i}$ or $E_{i}>E_{t h}$ is not eligible, then abstain from being $\mathrm{CH}$; Case 1: if $d_{l t h}<d<d_{i}$ and $E_{i}>E_{t h}$, then wait for a random time $T_{R}$.

Step 7: Return Step 3, and the operations loop until all CHs have been built.

The NCHR denotes that the MN wants to looking for near nodes to be CHs. $T_{R}$ denotes the random backoff time. Referring to $802.11 \mathrm{~b}$ Standard [9], $T_{R}=$ $2^{\tau} \cdot$ rand $\cdot\left[\left(E_{0}-E_{i}\right) / E_{i}\right](\tau=0,1,2, \ldots 7)$, and rand denotes a $(0,1]$ uniform distribution random variable. When $E_{i}$ of one node is larger than others, this means that its $T_{R}$ is smaller than others in statistical average and it will be superior to be $\mathrm{CH}$ statistically. NCHAM denotes that a node declare to be satisfy the condition to be a $\mathrm{CH}$. BM denotes that one node gives up to be a $\mathrm{CH}$ when it finds other nodes declaring to be $\mathrm{CH}$. FCHR denotes the $\mathrm{CH}$ wants to find new $\mathrm{CHs}$ at a distance. After the $\mathrm{CH}$ framework has been built, FCHR messages will get to BS ultimately. BS will calculate the total cost function values transmitted from near $\mathrm{CHs}$ and select the path with minimum cost value as the routing path. After that, BS will inform every $\mathrm{CH}$ on the routing path according to the ID routing order table.

Under the premise of the distance threshold $d_{s t h}$, we hope to find enough CNs with CSI good enough to participate in the cooperative MIMO transmission. The selection algorithm of CNs can be described as follows:

Step 1: $\mathrm{CN}$ request message(CNRM) is broadcasted by $\mathrm{CH}$. After CNRM is accepted by near sensor nodes. If $d<d_{s t h}$, then the sensor nodes are marked as pre-CNs, and transmit ACK messages and pilots to the $\mathrm{CH}$; otherwise, give up to be pre-CNs.

Step 2: The channel is estimated by $\mathrm{CH}$ according to the ACK messages and pilots, and the nodes of which CSI is good enough are defined as CNs. CN Acknowledge (CNACK) messages are transmitted to these CNs, and other pre-CNs which have not accepted CNACK message will sleep to save more energy.

\section{B. The Phase of Data Transmission}

After the $\mathrm{CH}$ framework and routing path have been built, MN sends its data packets to the near $\mathrm{CH}$, and it will encode the data packet and broadcast. Once the corresponding $\mathrm{CNs}$ receive the data packets, the $\mathrm{CH}$ and $\mathrm{CNs}$ will encode the data packet by orthogonal STBC, and transmit it in TDMA to the receiver in cooperative MIMO scheme. After receiving the packets, the $\mathrm{CH}$ in the next hop will decode them and try to correct the bit errors. If an error occurs after cooperative MIMO transmission, the $\mathrm{CH}$ of receiver will send a NACK message to the previous $\mathrm{CH}$ to request a retransmission. If the retransmission fails several times, the $\mathrm{CH}$ will return a message to tell the $\mathrm{MN}$ to re-build a new cost routing path. In cooperative MIMO transmissions, the STBC will be set by the antennas of transmitter and receiver. Since the sensor nodes are deployed randomly, we can not assure whether there are enough $\mathrm{CNs}$ around the $\mathrm{CH}$ or not. The CCEM [10] algorithm shows that if $\mathrm{CHs}$ can not find enough nodes, they can exchange their roles with other nearby nodes.

\section{The Phase of Routing Maintenance}

After a period of transmission on minimum cost path, the nodes on the path will consume energy dramatically, and the residual energy of these nodes will decrease quickly. If the location of the $\mathrm{MN}$ and the path from the $\mathrm{MN}$ to the BS do not change, the nodes on the path will be in death prematurely, which possibly makes the WSN not be a continuous network. Therefore, the routing must be refreshed periodically. The refreshing period may be determined by data forwarding cycles, and this forwarding cycles are determined by the size of statistical data bits gathered by MNs. We assume 
that a routing rebuilding happens after a data forwarding cycle in this paper.

During the data forwarding cycle, if the minimum cost path has been broken off suddenly by change, the WSN should have the function of self-healing in order to enhance the robustness of networks. When new $\mathrm{CHs}$ cannot be found to get to the BS, or data transmission fails after a pre-fixed number of retransmissions, the $\mathrm{MN}$ will be informed to rebuild the routing.

\section{COST FUNCTION ANALYSIS}

The cost function should be mainly in connection with such factors as energy consumption, residual energy of $\mathrm{CH}$, density of nodes and delay. The number of hops is a very important parameter for the cost function in cooperative MIMO transmissions, because the energy consumption and delay are mainly in connection with the total number of hops. When the total number of hops increases, the delay will increase proportionally and the total energy consumption will be affected [8]. When the sensor nodes with little residual energy act as the routing ones, the total cost value should be larger. On the other hand, the greater is the density of sensor nodes, the less is the cost value of these sensor nodes. In order to define and analyze the cost function of CRAT algorithm, we can analyze the energy consumption of the $j^{\text {th }}$ hop in cooperative MIMO transmission.

We assume $d_{\bar{m}_{-} T_{-} j}=d_{\bar{m}_{-} R_{-} j}=d_{\bar{m}}$ in CRAT, and the ILT cooperative MIMO transmission goes through a $k_{l}^{\text {th }}$-power path loss with additive white Gaussian noise (AWGN), and the IST SISO transmission goes through a $k_{s}^{\text {th }}$-power path loss with AWGN. Considering the effect of channel estimation, the total energy consumption per bit of the $j^{\text {th }}$ hop can be calculated by following formula:

$$
\begin{aligned}
& E_{b j}^{\text {mimo }}=\left[(1+a) \bar{E}_{b}^{\text {mimo }} \times \frac{(4 \pi)^{2} d_{j}^{k_{l}}}{G_{t} G_{r} \lambda^{2}} M_{l} N_{f}+\right. \\
& \left.P_{c}^{\text {mimo }} /\left(\rho R_{b}\right)\right]+\left(1+\sum_{i=1}^{N_{R_{-}-1}} L_{i}^{s t} / L\right) \times \\
& {\left[(1+a) \bar{E}_{b}^{\text {siso }} \times \frac{(4 \pi)^{2} d_{m}^{k_{s}}}{G_{t} G_{r} \lambda^{2}} M_{l} N_{f}+P_{c}^{s i s o} / R_{b}\right]}
\end{aligned}
$$

In (1), the total energy consumption per bit is equal to the sum of two terms. The first term denotes the energy consumption per bit in MIMO ILT transmission, and the second term denotes the energy consumption per bit in SISO IST transmission respectively in transmitter and receiver. Here, $\rho=\left(F-p N_{T_{-} j}\right) / F$, where $p N_{T_{-} j}$ is the pilots which are plugged in data block, and $p$ is the size of additional pilots of the data block transmitted by each antenna. $F$ is the size of data block determined by $F=\operatorname{floor}\left(T_{c} \times R_{s}\right)$, where $R_{s}$ is the symbol rate, and $T_{c}$ is the fading coherence time. $T_{c}$ can be estimated as $T_{c}=3 /\left(4 f_{m} \sqrt{\pi}\right)$, where the maximum Dopplershift $f_{m}$ is given by $f_{m}=v / \lambda$ with the velocity $v$ and the carrier wavelength $\lambda . L$ is the total of data bits transmitted in a data forwarding cycle in ILT or IST. $L_{i}^{s t}$ is the data bits of orthogonal STBC, which is determined by the size of constellation. $P_{c}^{\text {mimo }}$ and $P_{c}^{\text {siso }}$ denote the total circuit power except for the power amplifier for the cooperative MIMO and SISO transmission respectively. Other parameters are the same as shown in [2] and [11].

In (1), the average forwarding energy consumption per bit can be given by:

$$
{\overline{E_{b}}}^{\text {mimo }}=\frac{\gamma_{b} N_{T_{-} j} N_{0}}{\|H\|^{2}}=\frac{2 \gamma_{b} N_{T_{-j}} N_{0}}{\chi_{2 N_{T_{-} j} N_{R_{-} j}}^{2}}
$$

where, $H$ is the channel matrix, $\chi_{2 N_{T_{-j} j} N_{R_{-} j}}^{2}$ is a chi-squared random variable with degree of freedom $2 N_{T_{-} j} N_{R_{-j} j}$, and $\gamma_{b}$ is the signal to noise ratio (SNR) of the receiver. For BPSK, $\gamma_{b}=\left[Q^{-1}\left(P_{b}\right)\right]^{2} / 2$, where $Q^{-1}\left(P_{b}\right)$ denotes the antifunction of $Q(x)$ relating to BER $P_{b}$.

We can see from (1) and (2), when other parameters are fixed, the total energy consumption per bit of the cooperative MIMO transmission in the $j^{\text {th }}$ hop is mainly in connection with $d_{j}^{k_{l}}, d_{\frac{m_{s}}{m}}^{k_{1}}, N_{T_{-} j}, N_{R_{-} j}$. Therefore, the total energy consumption per bit can be given by:

$$
E_{b j}^{m i m o}=E_{b j}^{m i m o}\left(d_{j}^{k_{l}}, d_{m}^{k_{s}}, N_{T_{-} j}, N_{R_{-} j}\right)
$$

Obviously, the total energy consumption of the multi-hop cooperative MIMO transmission can be denoted as:

$$
E_{L}^{\text {mimo }}=L \cdot\left(E_{M C}^{s i s o}+\sum_{j=1}^{J} E_{b j}^{m i m o}-E_{B S}^{e l e c t}\right)
$$

where, $E_{M C}^{s i s o}$ denotes the total energy consumption in data transmitting from $\mathrm{MN}$ to the first $\mathrm{CH}, E_{B S}^{\text {elect }}$ denotes the energy saving of the last hop because of the BS whose energy is unlimited, and $J$ denotes the total number of hops.

If the transmission distance is $d_{j}$ in SISO transmission, then the total energy consumption per bit can be denoted as $E_{b j}^{s i s o}\left(d_{j}^{k_{l}}\right)$ given by [2]:

$$
E_{b j}^{s i s o}\left(d_{j}^{k_{l}}\right)=(1+a) \bar{E}_{b}^{s i s o} \times \frac{(4 \pi)^{2} d_{j}^{k_{l}}}{G_{t} G_{r} \lambda^{2}} M_{l} N_{f}+P_{c}^{s i s o} / R_{b}
$$

Here, the parameters are similar to the ones in cooperative MIMO transmission. Therefore, the condition of saving energy can be given by:

$$
E_{b j}^{m i m o}\left(d_{j}^{k_{l}}, d_{\frac{k_{s}}{m}}^{k_{1}} N_{T_{-} j}, N_{R_{-} j}\right) \leq E_{b j}^{s i s o}\left(d_{j}^{k_{l}}\right)
$$

When the equation premises hold, the value of $d_{j}$ is equal to the minimum ILT distance, i.e. the threshold $d_{l t h}$. When $d_{j}$ increases, energy can be saved in cooperative MIMO transmission.

From above analysis, we can see that with the routing distance increasing from $\mathrm{MN}$ to $\mathrm{BS}$, the total cost value will increase because the total energy consumption increases. On the other hand, if the total number of hops $J$ increases, the delay will increase too. The delay of the network will affect the cost value. Therefore, for some WSN where delay is very important, we should adjust the coefficient of hops to control the effect of delay in cost function. At the same time, the effects of residual energy and antennas are considered in the cost function. According to the routing tree structure of CRAT 
algorithm, we can see that the cost values of the CHs in every hops obey the principle of linear superposition. Therefore, the cost value of the receiver $\mathrm{CH}$ in the $j^{\text {th }}$ hop can be denoted as:

$$
C_{R_{-} j}=C_{T_{\_} j}+C_{j}
$$

where, $C_{j}$ denotes the cost of the $j^{\text {th }}$ hop from transmitter to receiver in cooperative MIMO transmission, and it can be defined as:

$$
C_{j}=\frac{L \cdot j^{\beta} E_{b j}^{m i m o}\left(d_{j}^{k_{l}}, d_{m}^{k_{s}}, N_{T_{\_} j}, N_{R_{\_} j}\right)}{\left(E_{T_{\_} j}+E_{R_{\lrcorner} j}\right)\left(N_{T_{\_} j}+N_{R_{\_} j}\right)}
$$

Here, $\beta$ is the coefficient of the hops $j$. With $\beta$ increasing, the importance of delay will be enhanced. Obviously, in order to obtain better sensor coverage and energy consumption balance, the nodes with lower residual energy level are more important than that with higher residual energy level, and sparser sensor nodes are more important than the denser sensor nodes too. Therefore, we hope the cost function value will increase with residual energy and density of the nodes decreasing. when $j=0$, it means the first hop from $\mathrm{MN}$ to the first $\mathrm{CH}$, then the total cost function from $\mathrm{MN}$ to $\mathrm{BS}$ is $C_{0}+C_{1}+, \ldots C_{j}, \ldots+C_{J}$, and it can be given by

$$
C_{\text {total }}=\frac{\sum_{j=0}^{J} L \cdot j^{\beta} E_{b j}^{m i m o}\left(d_{j}^{k_{l}}, d_{m}^{k_{s}}, N_{T_{\_} j}, N_{R_{\_} j}\right)}{\left(E_{T_{\_} j}+E_{R_{\_} j}\right)\left(N_{T_{\_} j}+N_{R_{\lrcorner} j}\right)}
$$

\section{EXPERIMENT}

To evaluate the reasonableness and effectiveness of the CRAT algorithm and the total cost function defined in (9), we assume that the sensor nodes are deployed uniformly and they are dense enough. The size of each data packet is 1000 bits. $N_{T} \times N_{R}$ Alamouti space-time code and BPSK modulation are adopted in each hop. The number of antennas in transmitter and receiver is $N_{T_{-} j}=N_{R_{-} j}=N_{T}=N_{R}$. Cooperative MIMO transmission goes through Rayleigh flat fading channels. Here, the total routing transmission distance in all experiments is $1000 \mathrm{~m}$ from MN to BS. The energy of every sensor node is divided into 10 levels, and all of the energy levels of nodes start from the $10^{\text {th }}$ level, and $E_{t h}$ is the first level. $L=L_{i}^{s t}=1000$ bits, $R_{b}=10 \mathrm{Kbps}, p=10, d_{l t h}=100 \mathrm{~m}$, $d_{s t h}=40 \mathrm{~m}, d_{i}=300 \mathrm{~m}, d_{\bar{m}_{-} T_{-} j}=d_{\bar{m}_{-} R_{-} j}=d_{\bar{m}}=20 \mathrm{~m}$ and other parameters setting such as $M_{l}, N_{f}$, are as the same as those in [2] and [11].

When the initial transmission starts under the premise of being with ILT thresholds or without ILT threshold, the total cost values from MN to BS in each data forwarding cycle in multi-hops transmission are shown in Fig. 3. Here, the energy levels of all sensor nodes are $10, \beta=0$, and $2 \times 2$ Alamouti space-time codes are used in all hops. From Fig. 3, we can see that, when thresholds are used in CRAT algorithm, the total energy consumption will decrease, and it leads the total cost value decrease. Here, the total cost values swarm in the range of $0.006 \sim 0.015$ approximately. However, if the thresholds are not used, the total cost values will swarm in the range of $0.008 \sim 0.02$ approximately. On the other hand, when the

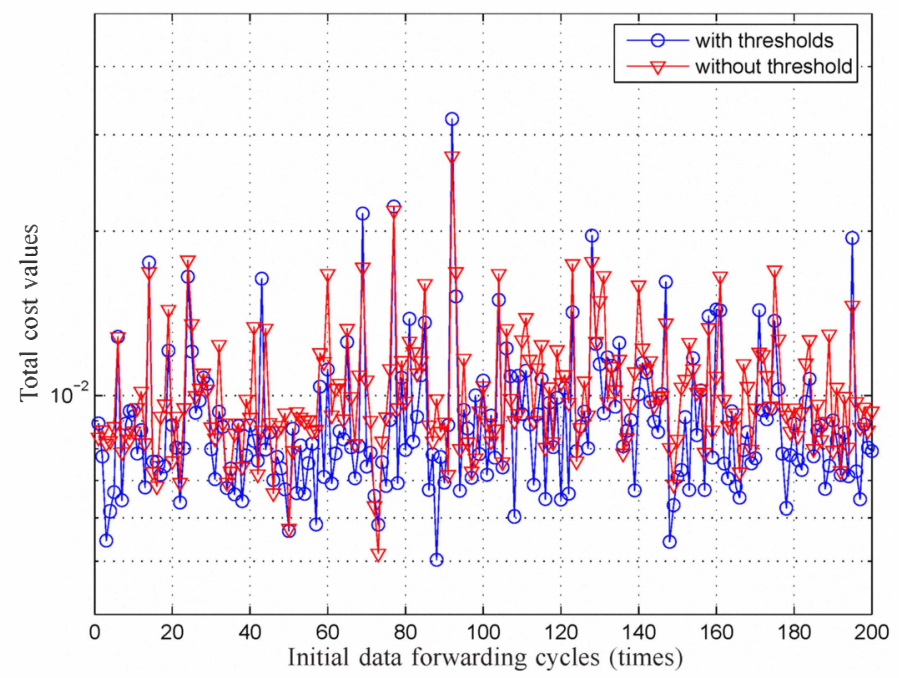

Fig. 3. Total cost values with and without threshold in initial data forwarding cycles

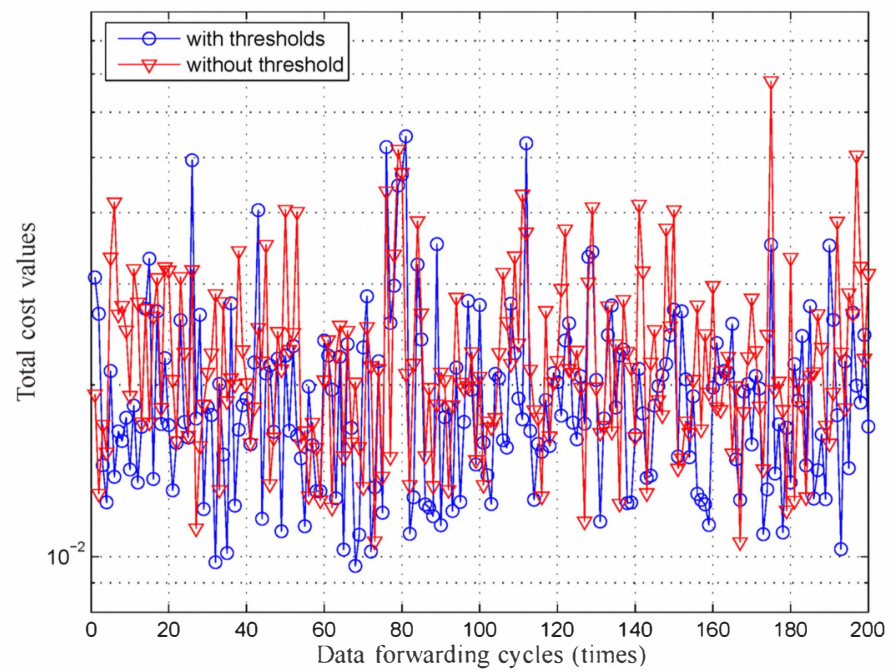

Fig. 4. Total cost values with and without threshold in data forwarding cycles

thresholds are used, the stability of the energy consumption is improved. With the energy of WSN consuming, the residual energy of every sensor node will become different. The total cost values in each data forwarding cycle will become larger than the total cost value in initial transmission. We assume the residual energy levels of every sensor nodes are randomly distributed. When the thresholds are used, the total cost values are smaller than that without threshold. The total cost values in each data forwarding cycle are shown in Fig. 4. From the figure, we can see that the statistical average cost values with thresholds are smaller than that without threshold. The results above show that CRAT algorithm is effective in reducing energy consumption and the total cost values.

The effect of the number of antennas (i.e., the total number of the CNs and their $\mathrm{CH}$ in each hop) is an important factor of cost values. For the CRAT algorithm, under conditions that $N_{T}=N_{R}=2, N_{T}=N_{R}=4$ and $N_{T}=N_{R}=8$, the total 


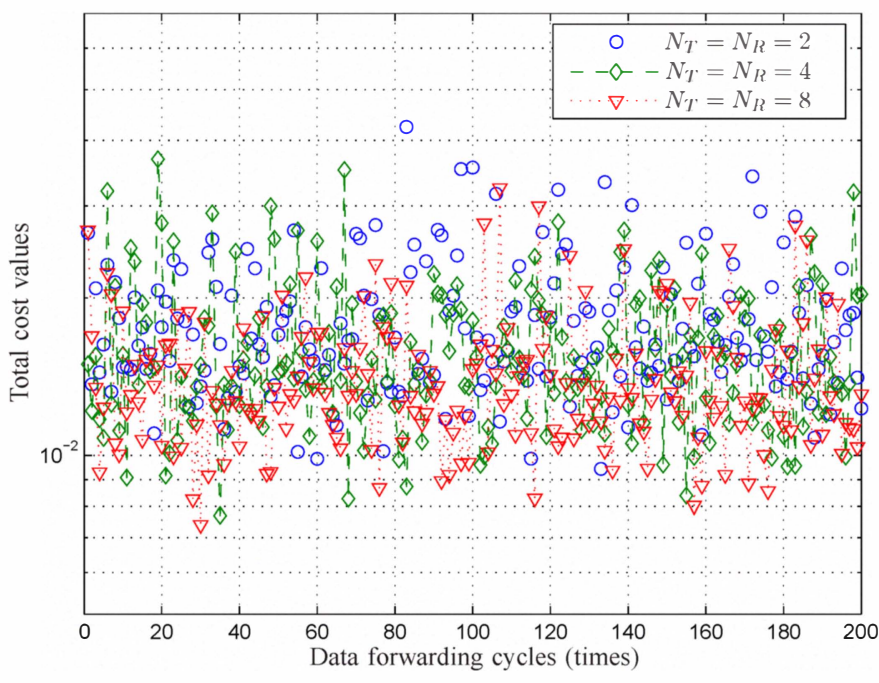

Fig. 5. Total cost values with different antennas in data forwarding cycles

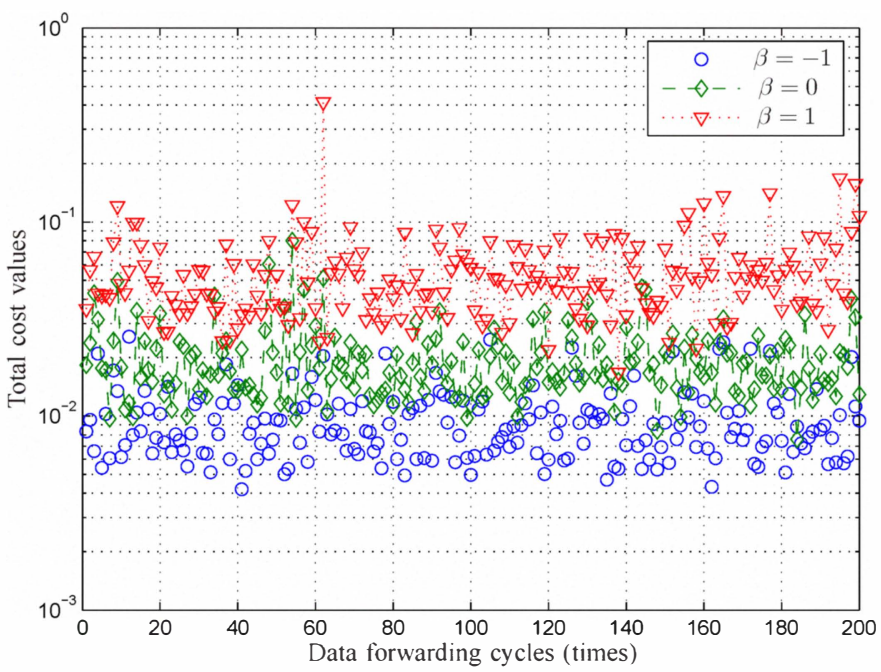

Fig. 6. Total cost values with different coefficient $\beta$ of hops in data forwarding cycles

cost values in each data forwarding cycle are shown in Fig. 5 . Since the residual energy of the sensor nodes are distributed randomly, the total cost values in the three circumstances swarm in the range of $0.01 \sim 0.03$, and only few values approach to 0.04 . We can see from the figure, the points of the total cost values are denser than any of the other two in the low position when $N_{T}=N_{R}=8$. This means that the BS would like to choose the path on which there are more sensor nodes to be CNs. Different value of $\beta$ will affect the distribution of total cost values. For example, Fig. 6 shows the total cost value under condition that $\beta=-1,0,1$. Here, in order to describe the relations of them, we fix the antennas on $N_{T}=N_{R}=2$. The upper points of cost value in the figure have more important delay performances than that lower points in the figure. In order to make a fair comparison, we should choose a suitable value of $\beta$ according to practical demand for delay. In a word, we can see that the cost function of CRAT algorithm is reasonable.

\section{CONCLUSION}

In this paper, we proposed a novel routing algorithm for multi-hop WSN based on cooperative MIMO. Unlike the other algorithms proposed before, we found and chose the thresholds as the step size to extend the networks in order to save more energy. Moreover, we analyzed the factors of cost routing and defined the cost function. Finally, the reasonableness of cost function and effectiveness of CRAT algorithm in saving energy were verified by experiments. The results show that the cost value will be reduced and its stability will be improved when the thresholds in CRAT are used. The future works will include the analysis of network life cycles, sensor coverage and complexity of WSN in CRAT algorithm with limited sensor nodes.

\section{ACKNOWLEDGMENT}

The project was supported by the National Natural Science Foundation of P.R. China $(60673086,60970041)$. X. Liu is the corresponding author.

\section{REFERENCES}

[1] I. F. Akyildiz, W. Su, Y. Sankarasubramaniam and E. Cayirci, "A survey on sensor networks," IEEE Communications Magazine, vol.40, pp. 102114, Aug. 2002.

[2] S. Cui, A. J. Goldsmith and A. Bahai, "Energy-efficiency of MIMO and cooperative MIMO techniques in sensor networks," IEEE Journal on Selected Areas in Communications, vol. 22, pp. 1089-1098, Aug. 2004.

[3] S.K. Jayaweera, "Virtual MIMO-based cooperative communication for energy-constrained wireless sensor networks," IEEE Transactions on Wireless Communications, Vol.5, pp. 984-989, May. 2006.

[4] W. Shi, J. Yuan and L. Lei, "Research on wireless sensor network coverage control algorithm," Acta Automatica Sinica, Vol. 35, pp. 540545, May. 2009.

[5] F. Ye, A. Chen, S. Lu and L. Zhang, "A scalable solution to minimum cost forwarding in large sensor networks," in Proc. ICCCN, 2001.

[6] D. Yuan, X. Liu, X. Zhang and H. Cho, "CEERP: Cost-based energyefficient routing protocol in wireless sensor networks," in Proc. APCCAS, 2008

[7] A. Aksu and O. Ercetin, "An energy-efficient routing protocol for networks with cooperative transmissions," in Proc. ICC'07, 2007.

[8] Y. Yuan, Z. He and M. Chen, "Virtual MIMO-based cross-layer design for wireless sensor networks," IEEE Transaction on Vehicular Technology, Vol. 55, pp. 856-864, May. 2006.

[9] IEEE Standard for Wireless LAN Medium Access Control (MAC) and Physical Layer (PHY) Specifications. ANSI/IEEE Standard 802.11, 1999.

[10] Q. Wang and $Y$. Qu, "An effective organizing method for wireless sensor networks based on MIMO" in Proc. WICOM, 2007.

[11] B. Zhao, J. Li, W. Zhang and Y. Qu, "MIMO-based energy-efficient wireless sensor networks," Acta Electronica Sinica, Vol. 34, pp. 14151419, Aug. 2006. 